\title{
La melatonina facilita la deshabituación a las benzodiazepinas en el insomnio
}

Facilitation of benzodiacepine discontinuation by melatonin. A new clinical approach. Garfinkel D, Zisapel N, Wainstein J et al. Arch Intern Med 1999;159:2456-2460.

\section{Objetivo}

Evaluar si la melatonina nocturna en el tratamiento del insomnio facilita la deshabituación a las benzodiazepinas (BZD).

\section{Diseño}

Estudio en dos períodos de seis semanas cada uno: 1) Ensayo clíniCo controlado y aleatorizado* (ECCA) doble ciego de melatonina vs placebo y 2) administración de melatonina simple ciego a todos los pacientes.

\section{Lugar}

Centro de Investigación geriátrica y Universidad de Tel Aviv, Israel.

\section{Pacientes}

Se enrolaron 34 pacientes que habían recibido BZD por al menos seis meses y estaban dispuestos a suspenderlas gradualmente.

\section{Intervención}

1) Los pacientes recibieron $2 \mathrm{mg}$ de melatonina (MT) de liberación controlada o placebo 2 hs antes de ir a dormir durante seis semanas. Se les solicitó reducir la dosis usual de BZD progresivamente: $50 \%$ la segunda semana, $75 \%$ la tercera y cuarta, y suspender completamente entre la quinta y la sexta. A los sujetos que no lograron reducir las BZD en el período uno se les solicitó el mismo esquema de reducción en el período dos.

\section{Medición de resultados principales}

Durante ambos períodos los participantes reportaban cada mañana la dosis usada de BZD y un puntaje de calidad de sueño (de 1 a 10) que fue promediado para cada semana del estudio. Solo los individuos que suspendieron completamente las BZD fueron considerados éxito del tratamiento.

El uso de BZD y la calidad de sueño fue reevaluada a los seis meses.

\section{Resultados Principales}

Ambos grupos fueron comparables al inicio en cuanto a sexo (70\% mujeres), edad (68+/- 16 años), tipo y dosis de BZD y calidad de sueño (puntaje 6.2 vs 6.8).

En el primer período, la tasa de suspensión de BZD fue superior en el grupo MT (14/18 vs $4 / 16)$ con una leve mejoría en el puntaje de sueño (no reportada).

En el segundo período, seis pacientes que habían fracasado con placebo lograron suspender las BZD sin deteriorar la calidad del sueño. Globalmente, de los 30 individuos que participaron en ambos períodos, 24 lograron suspender las BZD. A los seis meses, 79\% de ellos continuaban recibiendo MT sin requerir BZD y su puntjae de sueño fue superior al inicial. El éxito en la suspensión de BZD fue independiente al tipo específico de droga.

\section{Conclusión}

En pacientes con insomnio, la terapia con melatonina facilita la deshabituación a las BZD.

\section{COMENTARIO}

El insomnio es un trastorno definido por la dificultad para conciliar o mantener el sueño nocturno que compromete la actividad diurna habitual. Dado que puede afectar a casi el $20 \%$ de la población en algún momento de la vida, es un motivo muy frecuente de consulta en atención primaria. ${ }^{1}$ Es importante reconocer el tipo de insomnio según su duración (transitorio, a corto plazo o crónico) y realizàr una detallada evaluación de sus posibles causas, prestando especial atención a la depresión, el estrés post traumático, los trastornos por ansiedad generalizada y otros cuadros psicosociales en los que el insomnio no es más que otra manifestación dentro de un conjunto de signos y síntomas más definidos que tienen tratamientos específicos. ${ }^{2}$ Si bien los criterios de uso racional de BZD las recomiendan solo en el tratamiento inicial del insomnio transitorio y el de corto plazo, su indicación se ha extendido progresivamente al tratamiento del insomnio crónico y es hoy, lejos, el grupo de drogas más prescripto en todos los grupos etários, incluyendo a los ancianos. Las ventajas de las BZD son su eficacia, rapidez y relativa baja toxicidad. Pero es importante considerar que su uso crónico induce tolerancia, dependencia y abstinencia, así como alteraciones psicomotoras y cognitivas que se ven especialmente en ancianos, aún considerando la presencia de condiciones médicas crónicas. ${ }^{4}$ Este estudio nos presenta una alternativa interesante sobre la eficacia de la melatonina en el tratamiento del insomnio y su utilidad como coadyuvante en la deshabituación a las BZD. La MT fue útil para mantener una buena calidad de sueño en los pacientes que lograron suspenderla o disminuir drásticamente la dosis. La alteración que las BZD pueden inducir sobre el ritmo circadiano de la MT y la consecuente dificultad en la inducción del sueño en pacientes que presentan niveles mayores de MT diurna es una explicación razonable. La restauración de niveles elevados de MT durante la noche podría, entonces, ser responsable del efecto beneficioso sobre la calidad del sueño y de la posibilidad de suspender las BZD sin mayor efecto rebote. Uno de los interrogantes que quedan por resolver es durante cuánto tiempo se debe administrar la MT. Según los autores, hasta ahora no se ha reportado que el uso prolongado de MT haya inducido alteraciones cognitivas, tolerancia o abstinencia una vez suspendida, pero el tiempo de tratamiento no queda aún claro. Aparentemente, uno podría suspenderla una vez alcanzado un patrón de sueño estable y eventualmente reinstalarla si esto fallara.

Si bien, como todo ECCA, los participantes estaban claramente motivados, es también cierto que la mayoría de los pacientes manifiestan su deseo de suspender las BZD cuando esto se plantea en la consulta. Como dice la editorial, esto no disminuye la importancia del resultado alcanzado ya que la motivación, sumada al tratamiento, es la condición sine qua non para lograr la recuperación en una adicción.

\section{Dr. Fernando Rubinstein}

Unidad de Medicina Familiar. Hospital Italiano de Buenos Aires.

\section{Referencias}

1. Botargues M, Raze A. Ansiedad, depresión e insomnio en PROFAM: Programa de educacion a distancia de medicina familiar y ambulatoria / Rubinstein E, dir. Hospital Italiano de Bs As Departamento de docencia e investigación, c1997.

2. Melatonin Therapy: From benzodiazepine dependent insomnia to authenticity and autonomy. Arch intern med 1999;159: 2393-2395

3. Guidelines for the rational use of benzodiazepines. When and what to use. Drugs, 1994;48(1): 25-40.

4. Benzodiazepine exposure and functional status in older people. Journal of the American Geriatric Society, 1998;46:71-76 